Artículo original

\title{
Caracterización clínica de la leptospirosis grave en un hospital de alta complejidad de Cali, Colombia, 2010-2016
}

\author{
Jorge Cedano, Sarita Rodríguez ${ }^{1}$, Winy Kujundzic² ${ }^{2}$ Juan Sebastián Arana², Robinson \\ Pacheco', Fernando Rosso ${ }^{1,2,3}$ \\ ${ }^{1}$ Centro de Investigaciones Clínicas, Fundación Clínica Valle del Lili, Cali, Colombia \\ ${ }_{2}^{2}$ Programa de Medicina, Facultad de Ciencias de la Salud, Universidad Icesi, Cali, Colombia \\ ${ }^{3}$ Departamento de Medicina Interna-Infectología, Fundación Valle del Lili, Cali, Colombia
}

Introducción. La leptospirosis es una infección bacteriana endémica en Colombia. Su curso clínico puede ser variable y, en ocasiones, fatal. Hay pocos estudios en el país sobre los casos graves de esta enfermedad.

Objetivo. Describir las características demográficas y clínicas de los pacientes con diagnóstico de leptospirosis grave hospitalizados en salas generales o atendidos en la unidad de cuidados intensivos de un hospital de cuarto nivel.

Materiales y métodos. Se llevó a cabo un estudio observacional descriptivo de los pacientes adultos y niños con diagnóstico serológico de leptospirosis entre el 2010 y el 2016.

Resultados. Se analizaron las historias clínicas de 87 pacientes, $74 \%$ de los cuales correspondía a hombres y, el $84 \%$, a mayores de 18 años. El $35 \%$ tenía alguna comorbilidad y la hipertensión arterial sistémica (16\%) y la diabetes mellitus ( $9 \%$ ) fueron las más comunes. Los síntomas más frecuentes fueron: fiebre, náuseas, astenia, mialgias, artralgias y dolor abdominal. El 34 \% requirió atención en la unidad de cuidados intensivos, con una mediana de estancia de 5 días. El $61 \%$ requirió hospitalización en sala general, con una mediana de estancia de 6 días. Todos los casos recibieron tratamiento antibiótico con ceftriaxona o doxiciclina. La tasa de letalidad fue del 1,1\% $(n=1)$.

Conclusiones. La infección por Leptospira spp. tiene el riesgo de diagnosticarse de manera tardía por su presentación clínica inespecífica, lo que implica considerar un gran número de diagnósticos diferenciales. La atención temprana de los pacientes con cuadros graves de esta enfermedad en la unidad de cuidados intensivos, puede evitar una mayor incidencia de complicaciones y disminuir la mortalidad.

Citación:

Cedano J, Rodríguez S, Kujundzic W, Arana JS, Pacheco R, Rosso F. Caracterización clínica de la leptospirosis grave en un hospital de alta complejidad de Cali, Colombia, 2010-2016. Biomédica. 2019;39(Supl.1):108-16 https://doi.org/10.7705/biomedica.v39i2.3985

\section{Correspondencia:}

Fernando Rosso, Avenida Simón Bolívar, Carrera 98 № 18-49, Cali, Colombia

Teléfono: (316) 449 9673; fax: (572) 3317499

fernando.rosso@fvl.org.co

\section{Contribución de los autores:}

Winy Kujundzic, Juan Sebastián Arana y Sarita Rodríguez: recolección de datos y creación de base de datos

Fernando Rosso, Jorge Cedano y Robinson

Pacheco: análisis e interpretación de datos, revisión crítica del contenido

Todos los autores participaron en la redacción del manuscrito.

Financiación:

Este estudio fue financiado con fondos de la

Fundación Valle del Lili.

Conflicto de intereses:

Los investigadores declaran no tener conflictos de intereses.

Palabras clave: Leptospira; leptospirosis/diagnóstico; espiroquetas; enfermedad de Weil; factores de riesgo.

\section{Clinical characterization of patients with severe leptospirosis in a tertiary hospital in} Cali, Colombia, 2010-2016

Introduction: Leptospirosis is an endemic bacterial infection in Colombia. Its clinical course can be variable and occasionally fatal. There are few studies in the country about severe cases of leptospirosis.

Objectives: To describe the demographic and clinical characteristics of patients with a diagnosis of leptospirosis, and their management in a high complexity hospital.

Materials and methods: This was a descriptive retrospective study of patients with a serologic diagnosis of leptospirosis between 2010 and 2016.

Results: We analyzed 87 patients, $74 \%$ of them were men, and $84 \%$ were older than 18 years; $35 \%$ had a comorbidity, the most common being arterial hypertension (16\%) and diabetes mellitus (9\%). The most frequent symptoms were fever, nausea, fatigue, myalgia, arthralgia, and abdominal pain. The majority of patients required hospitalization in general wards $(61 \%)$, with a median stay of six days; $34 \%$ required management in the intensive care unit, with a median stay of five days. Mortality was $1.1 \%(n=1)$. All patients received treatment with either ceftriaxone or doxycycline.

Conclusions: There is a risk of leptospira infections having a late diagnosis given their unspecific clinical presentation, which generates a high number of differential diagnoses. The early management in the intensive care unit could decrease the incidence of complications and the mortality of patients with leptospirosis.

Keywords: Leptospira; leptospirosis/diagnosis; spirochaetales; Weil disease; risk factors. 
La leptospirosis es una zoonosis causada por especies patógenas de Leptospira (espiroquetas) del género L. interrogans sensu lato. Hasta ahora se han identificado, aproximadamente, 200 cepas, con una gran variabilidad antigénica (1).

La infección se adquiere por contacto con agua, alimentos o suelos contaminados con orina de animales infectados. Los reservorios más comunes son los roedores, las vacas, los cerdos, los perros, los caballos, las ovejas y las cabras $(2,3)$, que, usualmente, son portadores asintomáticos y cuyos túbulos renales son colonizados por la bacteria, la cual es excretada en la orina durante el resto de sus vidas (4).

La infección en los humanos se adquiere por contacto con fuentes contaminadas cuando hay abrasiones en la piel, las membranas mucosas o las conjuntivas, y raramente, por la ingestión de alimentos contaminados (2). Ciertos grupos de poblaciones tienen mayor riesgo de exposición debido a su trabajo en actividades agrícolas o en la manipulación de animales o de sus derivados (trabajadores de mataderos, militares, médicos veterinarios y auxiliares de clínicas veterinarias, ordeñadores en granjas de ganado, agricultores y operarios en instalaciones porcícolas o piscícolas) $(2,5)$.

La enfermedad es de distribución mundial, con mayor incidencia en los países tropicales debido a las condiciones climáticas que favorecen su transmisión. Es endémica en el Caribe, Latinoamérica, el subcontinente indio, Asia suroccidental, Oceanía y en zonas occidentales de Europa $(2,6)$.

La incidencia anual estimada es de 0,1 y 10 casos por 100.000 habitantes en climas templados y tropicales, respectivamente. En las zonas de brotes, como Brasil, el sudeste asiático y China, se reporta una incidencia de 100 por cada 100.000 habitantes (7). Se estima que se presentan 1'030.000 casos y 58.900 muertes al año a nivel mundial (8).

En Colombia, la prevalencia de esta zoonosis reemergente oscila entre el 6 y el $35 \%$ en humanos. Los departamentos del Valle del Cauca, Antioquia, Risaralda y Atlántico tienen el nivel más alto de notificación (7), la cual es obligatoria desde el 2007. La prevalencia en animales es del 41 al $61 \%$ en ganado bovino, del $10 \%$ en cerdos, del $47 \%$ en perros, del $23 \%$ en primates no humanos, y del 25 al $83 \%$ en roedores. Además, se asocia con actividades acuáticas en agua dulce y con condiciones de pobreza $(2,9)$.

Las infecciones por Leptospira spp. pueden ser asintomáticas o presentarse con signos y síntomas inespecíficos, como cefalea, mialgias, náuseas, vómitos, diarrea, erupción cutánea, dolor abdominal, eritema conjuntival y esplenomegalia. En casos graves, puede presentarse compromiso orgánico, choque séptico, dificultad respiratoria, miocarditis, rabdomiólisis, vasculitis, hemorragia, disfunción hepática o renal y muerte. Aproximadamente, el $10 \%$ de las infecciones sintomáticas se presenta como enfermedad de Weil, la cual afecta los riñones y el hígado (2-4). Por su presentación inespecífica, el diagnóstico se basa en la sospecha clínica y la presencia de factores de riesgo, y puede confirmarse con la serología. Se trata con antibióticos como doxicilina, penicilina $\mathrm{G}$ o ceftriaxona, dependiendo de la gravedad de la infección $(6,9)$.

En la actualidad, el diagnóstico de leptospirosis es un reto, ya que se requiere un alto grado de sospecha para detectarla, debido a que la presentación clínica es inespecífica y similar a la de otras enfermedades infecciosas. Por otro lado, las pruebas diagnósticas que se utilizan son de bajo rendimiento. La 
sensibilidad del cultivo y de la prueba de aglutinación microscópica (Microscopic Agglutination Test, MAT) solo alcanzan el $10 \%$ y el $49 \%$, respectivamente; si se practican las dos, la sensibilidad se incrementa apenas al $55 \%$ (10).

En los últimos años, se ha fortalecido la vigilancia de la leptospirosis en Colombia y se ha establecido la notificación rutinaria por parte de las entidades territoriales. Sin embargo, en la literatura médica nacional hay pocos reportes sobre el curso clínico de esta enfermedad.

El propósito de este estudio fue describir el curso clínico y su resultado en pacientes con leptospirosis grave en un hospital de alta complejidad de Cali.

\section{Materiales y métodos}

Se llevó a cabo un estudio observacional descriptivo de una serie de pacientes tratados en un centro de cuarto nivel de complejidad de Cali entre enero del 2010 y junio del 2016.

Se incluyeron pacientes de ambos sexos, de todas las edades, con sospecha clínica de leptospirosis y prueba positiva de inmunoglobulina M (IgM ELISA-PanBio ${ }^{\circledR}$, BioMérieux) para leptospira, aunque sin confirmación con otras pruebas, como la MAT. Se solicitaron las historias clínicas y las fichas epidemiológicas al comité de vigilancia epidemiológica. Se registraron los datos sociodemográficos, las comorbilidades, las manifestaciones clínicas, los exámenes de laboratorio, el tratamiento y el resultado en cada caso.

Toda la información clínica, de laboratorio y epidemiológica, se recolectó en una base electrónica de datos. Se clasificaron las comorbilidades existentes antes del desarrollo de la infección, como hipertensión arterial sistémica, diabetes mellitus, insuficiencia renal crónica, cirrosis, enfermedad pulmonar obstructiva crónica e insuficiencia cardiaca congestiva, entre otras, a partir de los diagnósticos registrados en la historia clínica.

Se hizo el análisis univariado para evaluar la distribución de las variables numéricas mediante la prueba de Shapiro-Wilk. Los datos se resumieron usando medidas de tendencia central (promedios y desviación estándar) o mediana y rangos intercuartílicos, según correspondiera. Las variables cualitativas se expresaron como proporciones. Los análisis se hicieron con el paquete estadístico Stata $^{\mathrm{TM}}$ (Stata Corp, 2011, College Station, TX, USA).

El estudio tuvo limitaciones al tratarse de un estudio retrospectivo, en el cual los casos no fueron confirmados mediante MAT ni se determinaron los serovares.

\section{Consideraciones éticas}

El proyecto fue aprobado por el Comité de Ética de Investigaciones en Humanos de la Fundación Clínica Valle del Lili, según Acta No. 9 del 20 de abril de 2015.

\section{Resultados}

Entre enero de 2010 y junio de 2016, se analizaron las historias clínicas de 87 pacientes. La mediana de edad fue de 36 años (rango: 20-50); el $16 \%$ era menor de 18 años, con una mediana de edad de 13,5 (rango: 10-15), y los mayores de 18 años tenían una mediana de 38 años (rango: 26-57). El $73 \%$ de los pacientes eran hombres, con una mediana de edad de 34 años (rango: 20-40), en tanto que las mujeres tenían una mediana de 38 años (rango: 24-61). El $9 \%$ de los pacientes era mayor de 65 años. 
El $81 \%$ reportó contacto con animales, el $26 \%$ tuvo contacto con perros, el $20 \%$ lo tuvo con gatos, el $16 \%$ con roedores y, un porcentaje menor tuvo contacto con ganado bovino (3,5\%), equino (2,3\%) o porcino, y solo el $6 \%$ reportó que consideraba que estos animales estaban enfermos.

La fuente principal de agua en la mayoría de los casos era el acueducto $(40,2 \%)$ y solo el $2 \%$ reportó inundación reciente.

El $35 \%$ de los pacientes sufría enfermedad crónica, y las más comunes fueron la hipertensión arterial sistémica (16\%) y la diabetes ( $9 \%)$, en tanto que, en menor proporción, se reportó hipotiroidismo (6\%), falla renal crónica (4\%), cáncer (2\%) y cardiopatía (2\%) (cuadro 1).

Los síntomas más frecuentes fueron: fiebre (85\%), náuseas (67\%), astenia (49\%), mialgias (57\%), artralgias (64\%), dolor abdominal (52\%)

Cuadro 1. Casos de leptospirosis según características sociodemográficas, Fundación Valle del Lili, Cali, 2010-2016

\begin{tabular}{lcc}
\hline Variable & n (\%) \\
\hline Sexo masculino & 64 & $(73,56)$ \\
$0-17$ años & 14 & $(16,1)$ \\
$18-65$ años & 65 & $(74,7)$ \\
Desempleado & 15 & $(17,2)$ \\
Agricultor & 9 & $(10,3)$ \\
Militar & 8 & $(9,2)$ \\
Contacto con animales & 71 & $(81,6)$ \\
Contacto con ratas & 14 & $(16,1)$ \\
Agua de acueducto & 35 & $(40,2)$ \\
Embarazo & 5 & $(5,8)$ \\
Comorbilidades & 31 & $(35,6)$ \\
Hipertensión arterial sistémica & 14 & $(16,1)$ \\
Diabetes mellitus & 8 & $(9,2)$ \\
\hline
\end{tabular}

y diarrea (35\%). Las manifestaciones pulmonares fueron más comunes en adultos (20\%). En menores de 18 años, los síntomas más frecuentes fueron: fiebre (92\%), náuseas y dolor abdominal, con $71 \%$ cada uno. Las complicaciones hemorrágicas, como sangrado gastrointestinal (hematemesis o melenas) y epistaxis, fueron más comunes en los niños (28\%), en tanto que solo el $13 \%$ de los adultos las presentaron; se reportó hematuria en 5 pacientes y sangrado gastrointestinal en 4 . La diarrea fue una manifestación más común en niños (64 Vs. $30 \%$ ), y esta diferencia fue estadísticamente significativa (cuadro 2).

Las alteraciones más comunes detectadas en los exámenes de laboratorio, fueron: neutrofilia (55\%), anemia (52\%), leucocitosis (45\%), trombocitopenia (45\%), y elevación de la lactato deshidrogenasa (LDH) (39\%), de la bilirrubina total (42\%), de la alanino transaminasa (ALT) (38 $\%$ ) y de la aspartato aminotransferasa (AST) (39\%). El valor promedio de la ALT fue de $85 \mathrm{UI} / \mathrm{L}$ (desviación estándar, $\mathrm{DE}=38,4-159$ ), de la AST, 85,7 $\mathrm{UI} / \mathrm{L}$ ( $\mathrm{DE}=33-166)$, de la LDH, 350UI/L ( $\mathrm{DE}=245-636)$, de la bilirrubina total, $1,2 \mathrm{mg} / \mathrm{dl}(\mathrm{DE}=0,5-8,56)$ y de la bilirrubina directa, $0,56 \mathrm{mg} / \mathrm{dl}(\mathrm{DE}=0,16-$ 7). Además, 14 pacientes presentaron elevación de la ALT o la AST mayor 
Cuadro 2. Casos de leptospirosis según características clínicas, Fundación Valle del Lili Cali, 2010-2016

\begin{tabular}{|c|c|c|c|c|c|}
\hline Signos clínicos & & $\begin{array}{l}\text { otal } \\
\text { l=87) } \\
(\%)\end{array}$ & $\begin{array}{c}<18 \text { años } \\
(n=14) \\
n(\%)\end{array}$ & $\begin{array}{c}>18 \text { años } \\
(n=73) \\
n(\%)\end{array}$ & $\mathbf{p}$ \\
\hline Fiebre & 74 & $(85,1)$ & $13(92,9)$ & $61(83,6)$ & 0,372 \\
\hline Náuseas & 59 & $(67,8)$ & $10(71,4)$ & $49(67,1)$ & 1 \\
\hline Vómito & 27 & (31) & $6 \quad(42,9)$ & $21(28,8)$ & 0,297 \\
\hline Cefalea & 35 & $(40,2)$ & $(42,9)$ & $29(39,7)$ & 0,827 \\
\hline Escalofríos & 17 & $(19,5)$ & $(14,3)$ & $15(20,5)$ & 0,728 \\
\hline Exantema & 8 & $(9,2)$ & $(7,1)$ & $7 \quad(9,6)$ & 1 \\
\hline Astenia & 43 & $(49,4)$ & $(50)$ & $36(49,3)$ & 0,963 \\
\hline Mialgias & 50 & $(57,5)$ & $(28,6)$ & $46(63)$ & 0,017 \\
\hline Artralgias & 56 & $(64,4)$ & $7 \quad(50)$ & $49(67,1)$ & 0,238 \\
\hline Dolor abdominal & 46 & $(52,9)$ & $10(71,4)$ & $36(49,3)$ & 0,129 \\
\hline Diarrea & 31 & $(35,6)$ & $(64,3)$ & $22(30,1)$ & 0,03 \\
\hline Linfadenopatías & 7 & (8) & $(14,3)$ & $5 \quad(6,8)$ & 0,313 \\
\hline Ictericia & 24 & $(27,6)$ & $(7,1)$ & $23(31,5)$ & 0,1 \\
\hline Hepatomegalia & 11 & $(12,6)$ & $(14,3)$ & $9 \quad(12,3)$ & 1 \\
\hline Esplenomegalia & 3 & $(3,4)$ & 0 & $3 \quad(4,1)$ & 1 \\
\hline Manifestaciones pulmonares & 16 & $(18,4)$ & $(7,1)$ & $15(20,5)$ & 0,089 \\
\hline Hemorragia & 14 & $(16,1)$ & $(28,6)$ & $10(13,7)$ & 0,228 \\
\hline
\end{tabular}

de $400 \mathrm{UI} / \mathrm{L}$ y 7 presentaron elevación de la LDH a más de $1.000 \mathrm{UI} / \mathrm{L}$; en el análisis de orina de 16 de los pacientes se informaron alteraciones, especialmente hematuria (15\%) y proteinuria (11\%) (cuadro 3).

El $34 \%$ de los pacientes tuvo que ser atendido en la unidad de cuidados intensivos y, de este porcentaje, el $42 \%$ tenía alguna comorbilidad. La mediana de estancia hospitalaria fue de 6 días en las salas de hospitalización general (rango intercuartílico, $\mathrm{Rl}=4-9,5$ ) y, de 5 días, en la unidad de cuidados intensivos (RI=3-13), y 13 pacientes recibieron transfusión de hemoderivados. Solo hubo un caso fatal.

Los antibióticos más usados para el tratamiento de la leptospirosis fueron la ceftriaxona y la doxiciclina. Se presentaron lesiones orgánicas en 23 pacientes, y el $2 \%$ tuvo infecciones asociadas con la atención en salud. El $17 \%$ presentó enfermedad de Weil (ictericia, lesión renal con oliguria, síndrome de reacción inflamatoria sistémica y manifestaciones hemorrágicas), que requirió asistencia respiratoria mecánica en la unidad de cuidados intensivos (cuadro 4). Entre los pacientes que requirieron atención en esta unidad, la causa más frecuente de ingreso fue la falla renal aguda $(n=15)$, seguida del choque séptico $(n=2)$, la encefalopatía hepática $(n=2)$ y la miocarditis $(n=1)$.

\section{Discusión}

Se describen 87 pacientes con diagnóstico de leptospirosis atendidos en un centro de cuarto nivel de complejidad de Cali, Colombia. La mayoría de los pacientes eran hombres, hallazgo comparable con el de otros estudios nacionales (11), lo cual podría explicarse por la asociación entre el sexo y el tipo de actividades relacionadas con esta enfermedad (12,13). Además, el grupo de edad más frecuente fue el de los adultos en edad productiva. 
La presentación clínica de la leptospirosis tiene un amplio espectro. Las manifestaciones clínicas más frecuentes fueron: fiebre, náuseas, mialgia, dolor abdominal, artralgias, astenia y cefalea, cuadro clínico similar al reportado en otras series en las que la fiebre, las mialgias y el dolor abdominal habían sido los síntomas más comunes $(11,14)$. Otras manifestaciones, como la esplenomegalia, variaron significativamente entre los estudios (3-94 \%) (14).

Cuadro 3. Exámenes de laboratorio de pacientes con diagnóstico de leptospirosis

\begin{tabular}{|c|c|c|c|}
\hline Exámenes de laboratorio & Total (\%) & Exámenes de laboratorio & Total (\%) \\
\hline Leucocitosis (>11.000 células $/ \mathrm{mm}^{3}$ ) & $39(44,8)$ & Leucocituria & $8(0,2)$ \\
\hline Leucocitos (células $/ \mathrm{mm}^{3}$ ) & $10.455(7.950-15.150)$ & Hematuria & $13(14,9)$ \\
\hline Neutrofilia (>6.000 células $/ \mathrm{mm}^{3}$ ) & $48(55,2)$ & ALT valor & $85(8,4-159)$ \\
\hline Neutrófilos (células/mm³) & $7.150(3.600-11.670)$ & ALT>120 UI/L & $33(37,9)$ \\
\hline Hemoglobina (g/dl) & $10,7 \pm 2,9$ & $\mathrm{ALT}>400 \mathrm{UI} / \mathrm{L}$ & $5(5,7)$ \\
\hline Anemia (<12 g/dl) & $45(51,7)$ & AST & $85,7(33-166)$ \\
\hline Trombocitopenia $\left(<150.000 / \mathrm{mm}^{3}\right)$ & $39(44,8)$ & AST>120 UI/L & $34(39,1)$ \\
\hline Plaquetas (células $/ \mathrm{mm}^{3}$ ) & $163.000(111.000-237.000)$ & AST $>400 \mathrm{UI} / \mathrm{L}$ & $9(10,3)$ \\
\hline Hematocrito elevado (>45 \%) & $5(5,7)$ & $\mathrm{LDH}$ & $350(\mathrm{DE}=245-636)$ \\
\hline Hematocrito (\%) & $39(3-41)$ & $\mathrm{LDH}>200 \mathrm{UI} / \mathrm{L}$ & $40(46)$ \\
\hline Hipernatremia (>145 mEq/L) & $9(10,3)$ & $\mathrm{LDH}>1.000 \mathrm{UI} / \mathrm{L}$ & $7(8)$ \\
\hline Hiponatremia (<135 mEq/L) & $17(19,5)$ & Bilirrubina total (UI/L) & $1,2(0,5-8,56)$ \\
\hline Hiperpotasemia (>5,5 mEq/L) & $2(2,3)$ & Bilirrubina total elevada (>1,0 UI/L) & $37(42,5)$ \\
\hline Hipopotasemia (<3,5 mEq/L) & $8(9,2)$ & Bilirrubina directa (UI/L) & $0,56(0,16-7)$ \\
\hline Alteraciones en análisis de orina & $16(18,4)$ & Bilirrubina directa elevada $(>0,3 \mathrm{UI} / \mathrm{L})$ & $41(47,1)$ \\
\hline Proteinuria & $10(11,5)$ & & \\
\hline
\end{tabular}

ALT: alanino transaminasa; AST: aspartato aminotransferasa; LDH: lactato deshidrogenasa

Cuadro 4. Tratamiento y resultados en pacientes con diagnóstico de leptospirosis, Fundación Valle del Lili, Cali, 2010-2016

\begin{tabular}{lrrr}
\hline Variables & $\begin{array}{c}\text { Total } \\
\mathbf{( N = 8 7 )} \\
\mathbf{n}(\%)\end{array}$ & $\begin{array}{c}<18 \text { años } \\
(\mathbf{n = 1 4 )} \\
\mathbf{n}(\%)\end{array}$ & \begin{tabular}{c}
\multicolumn{1}{c}{$\mathbf{1 8}$ años } \\
$\mathbf{( n = 7 3 )}$ \\
$\mathbf{n}(\%)$
\end{tabular} \\
\hline Urgencias & $73(83,9)$ & $12(85,7)$ & $61(83,6)$ \\
Ambulatorio & $1(1,1)$ & 0 & $1(1,4)$ \\
Hospitalización & $47(54)$ & $9(64,3)$ & $38(52,1)$ \\
Urgencias & $9(10,3)$ & $1(7,1)$ & $8(11)$ \\
Unidad de cuidados intensivos & $29(33,3)$ & $4(28,6)$ & $25(34,2)$ \\
Presencia de choque & $8(9,2)$ & $1(7,1)$ & $7(9,6)$ \\
Días de hospitalización & $6(4-9,5)$ & $7(3-9)$ & $6(4-10)$ \\
Días en la unidad de cuidados & $5(3-13)$ & $6(2,5-12)$ & $5(3-13)$ \\
intensivos & & & \\
Transfusiones & & & \\
Glóbulos rojos & $12(13,8)$ & $3(21,43)$ & $9(12,33)$ \\
Plaquetas & $3(3,4)$ & $0(0)$ & $3(4,11)$ \\
Plasma & $2(2,3)$ & $0(0)$ & $2(2,74)$ \\
Uso de antibióticos & $76(87,4)$ & $12(85,71)$ & $64(87,67)$ \\
$\quad$ Días de uso & $9(6-10)$ & $9(8,5-10,5)$ & $7(5-10)$ \\
Uso de vasopresores & $4(4,6)$ & $1(7,14)$ & $3(4,11)$ \\
Muerte & $1(1,1)$ & 0 & $1(1,4)$ \\
\hline
\end{tabular}


Es importante resaltar que la frecuencia de ictericia fue menor que la reportada en otros estudios (45,5 a $62 \%$ ). Este signo puede encontrarse en un gran porcentaje de los pacientes que fallecen, como una de las manifestaciones del síndrome de Weil, forma de presentación grave de la enfermedad (11). Esta menor proporción de ictericia pudiera sugerir que los casos fueron detectados más tempranamente.

En el presente estudio, no se utilizó la prueba de referencia (MAT) para el diagnóstico de la leptospirosis ya que en Colombia, habitualmente, se utiliza con propósitos de vigilancia y su disponibilidad es limitada, por lo que solo se emplea en centros especializados. Por ello, el diagnóstico se basó en la sospecha clínica y la detección de los anticuerpos (IgM) de leptospira.

En cuanto a los resultados de los exámenes adicionales de laboratorio, la mayoría de los pacientes presentaron neutrofilia y, cerca de la mitad, trombocitopenia, lo cual se ha descrito en casos de leptospirosis grave en los que hasta el $50 \%$ puede presentar trombocitopenia $(15,16)$.

Los diagnósticos diferenciales de cuadros con manifestaciones similares a la leptospirosis, son múltiples y se asocian principalmente con infecciones como el dengue y el chikungunya. En este estudio, se documentó un solo caso de infección concomitante con dengue, el cual no requirió atención en la unidad de cuidados intensivos, se trató con antibióticos y tuvo un curso clínico benigno. En presencia de estas infecciones virales concomitantes, el tratamiento antibiótico oportuno puede reducir significativamente la mortalidad $(17,18)$.

La mortalidad entre los pacientes atendidos en nuestra institución, está por debajo de lo reportado en el Sistema Nacional de Vigilancia en Salud Pública de Colombia. La mortalidad descrita varía entre 1,5 y $5 \%$, e incluso, es mayor en casos graves, hasta alcanzar el $10 \%$ (15). En informes recientes de la literatura médica sobre casos atendidos en Colombia, se reportó una mortalidad del $5 \%$ (11), mayor que la registrada en este estudio ( 5 Vs. $1 \%$ ); el porcentaje de pacientes con síndrome de Weil también fue menor en la presente serie de casos (17 Vs. $38 \%$ ), así como la duración de la hospitalización en cuidados intensivos (34 Vs. $16 \%$ ). Estas diferencias en la mortalidad podrían estar asociadas con el reconocimiento tardío de la enfermedad, la falta de un tratamiento médico agresivo o la dificultad de acceso a las unidades de cuidados intensivos. En diversos estudios publicados, se ha concluido que es preferible atender los casos graves en este tipo de unidades $(11,19,20)$.

Es difícil determinar tempranamente la necesidad de atención en la unidad de cuidados intensivos de estos pacientes. Complicaciones como la falla renal aguda que requiere diálisis, la diátesis hemorrágica y la ictericia grave, son las más comúnmente reportadas como asociadas con la leptospirosis grave, lo que denota un diagnóstico tardío de la enfermedad (19) y de las complicaciones, y ello, a su vez, estaría asociado con una mayor mortalidad. El retraso en el reconocimiento y en el tratamiento de estos pacientes puede tener resultados graves, como insuficiencia renal, insuficiencia hepática e, incluso, la muerte (20).

La presentación inespecífica de la leptospirosis dificulta el diagnóstico temprano, especialmente, por su sintomatología similar a la de otros síndromes febriles en áreas endémicas. Por lo tanto, se debe hacer énfasis en el diagnóstico más temprano de la infección y de sus complicaciones. 
En conclusión, la presente serie incluyó casos complejos de la enfermedad, aunque el diagnóstico y el reconocimiento temprano de la enfermedad y de sus complicaciones, así como un mayor acceso a los cuidados intensivos, se asociaron con una menor mortalidad. Estos hallazgos sugieren que la mortalidad por leptospirosis es prevenible.

\section{Referencias}

1. Chin J. Manual de Control de Enfermedades Transmisibles. $16^{\underline{a}}$ edición. Washington, D.C.: Organización Panamericana de la Salud; 1997. p. 564-5.

2. Day N. Epidemiology, microbiology, clinical manifestations and diagnosis of leptospiroris, 2017. Fecha de consulta: 2 de mayo de 2017. Disponible en: http://www.uptodate.com/ contents/epidemiology-microbiology-clinical-manifestations-and-diagnosis-of-leptospirosis

3. Dupouey J, Faucher B, Edouard S, Richet H, Broucker A, Marie L, et al. Epidemiological investigation of a human leptospirosis case reported in a suburban area near Marseille. New Microbes New Infect. 2014;2:82-3 https://doi.org/10.1002/nmi2.45

4. Murray PR. Microbiología médica. Sexta edición. Madrid: Elsevier; 2009. p. 416-9.

5. Góngora A, Parra J, Aponte L, Gómez L. Seroprevalencia de Leptospira spp. en grupos de población de Villavicencio, Colombia. Rev Salud Pública (Bogotá). 2008;10:269-78. https://doi.org/10.1590/S0124-00642008000200007

6. de Vries SG, Visser BJ, Nagel IM, Goris MG, Hartskeerl R, Grobusch MP. Leptospirosis in Sub-Sahara Africa: A systematic review. Int J Infect Dis. 2014;28:47-64. https://doi.org/10.1016/j.ijid.2014.06.013

7. Bello S, Rodríguez M, Paredes A, Mendivelso F, Walteros D, Rodríguez F, et al. Comportamiento de la vigilancia epidemiológica de la leptospirosis humana en Colombia 2007-2011. Biomédica. 2013;33(Supl.1):1608. https://doi.org/10.7705/biomedica.v33i0.1608

8. Costa F, Hagan JE, Calcagno J, Kane M, Torgeson P, Martínez MS, et al. Global morbidity and mortality of leptospirosis: A systematic review. PLos Negl Trop Dis. 2015;17:9. https://doi.org/10.1371/journal.pntd.0003898

9. Carreño LA. Prevalencia de leptospirosis en Colombia. Revisón sistemática de la literatura (tesis). Bogotá: Universidad Nacional de Colombia; 2014.

10. Limmathurotsakul D, Turner EL, Wuthiekanun V, Thaipadungpanit J, Suputtamongkol $Y$, Chierakul W, et al. Fool's gold: Why imperfect reference test are undermining the evaluation of novel diagnostics: A reevaluation of a 5 diagnostic tests for leptospirosis. Clin Infect Dis. 2012;55:322-31. https://doi.org/10.1093/cid/cis403

11. Echeverri-Toro LM, Penagos S, Castañeda L, Villa P, Atehortúa S, Ramírez F, et al. Características sociodemográficas y clínicas de pacientes con infección por Leptospira spp. atendidos en cuatro centros hospitalarios de Medellín, Colombia 2008-2013. Biomédica. 2017;37:62-7. https://doi.org/10.7705/biomedica.v37i1.3280

12. Ferro BE, Rodríguez AL, Pérez M, Travi BL. Seroprevalencia de infección por Leptospira en habitantes de barrios periféricos de Cali. Biomédica. 2006;26:250-7. https://doi.org/10.7705/biomedica.v26i2.1414

13. García R, Reyes A, Basilio D, Ramírez M, Rivas B. Leptospirosis: un problema de salud pública. Rev Latinoamer Patol Clin. 2013;60:57-70.

14. Pérez-García J, Arboleda M, Agudelo-Flórez P. Leptospirosis infantil en pacientes con síndrome febril en la región de Urabá, Colombia. Rev Peru Med Exp Salud Pública. 2016;33:745-50. https://doi.org/10.17843/rpmesp.2016.334.2561

15. Cruz CM, Arboleda M. Perfil hematológico, renal y hepático en pacientes con leptospirosis. Primera edición. Medellín: CES; 2011.

16. Rojas G, Kong J, Donoso A, Prado P. Una causa infrecuente de falla renal aguda e ictericia. Leptospirosis: caso clínico y revisión de la literatura. Rev Chil Pediatr. 2001;72:230-4. https://doi.org/10.4067/S0370-41062001000300008

17. Wijesinghe A, Gnanapragash N, Ranasinghe G, Ragunathan MK. Fatal co-infection with leptospirosis and dengue in a Sri Lankan male. BMC Res Notes. 2015;8:348. https://doi.org/10.1186/s13104-015-1321-7 
18. Nhan TX, Bonnieux E, Rovery C, De Pina JJ, Musso D. Fatal leptospirosis and chikungunya co-infection: Do not forget leptospirosis during chikungunya outbreaks. IDCases. 2016;5:12-4. https://doi.org/10.1016/j.idcr.2016.06.003

19. Vieira S, Brauner J, Fonseca D. Leptospirosis in the intensive care unit: A cohort of 57 patients. Crit Care. 2005;9(Suppl.1):31. https://doi.org/10.1186/cc3094

20. Michalopoulos A, Pappas G, Papadakis E, Christoforatos T, Malamos P, Koumoudiou C, et al. Leptospirosis in a European intensive care unit. Scand J Infect Dis. 2010;42:69-71. https://doi.org/10.3109/00365540903302861 